Itinéraires Itinéraires

Littérature, textes, cultures

2012-2 | 2012

Intime et politique

\title{
Conditions et formes d'une sociabilité littéraire chez Maurice Blanchot
}

\section{Cyril Coubard}

\section{(2) OpenEdition}

1 Journals

Édition électronique

URL : http://journals.openedition.org/itineraires/1177

DOI : 10.4000/itineraires. 1177

ISSN : 2427-920X

Éditeur

Pléiade

\section{Édition imprimée}

Date de publication : 1 novembre 2012

Pagination : 143-160

ISBN : 978-2-336-00027-5

ISSN : $2100-1340$

\section{Référence électronique}

Cyril Coubard, « Conditions et formes d'une sociabilité littéraire chez Maurice Blanchot », Itinéraires [En ligne], 2012-2 | 2012, mis en ligne le 01 novembre 2012, consulté le 10 décembre 2020. URL : http:// journals.openedition.org/itineraires/1177; DOI : https://doi.org/10.4000/itineraires.1177

\section{(c) $(1) \&$}

Itinéraires est mis à disposition selon les termes de la licence Creative Commons Attribution - Pas d'Utilisation Commerciale - Pas de Modification 4.0 International. 


\title{
Conditions et formes d'une sociabilité littéraire chez Maurice Blanchot
}

\begin{abstract}
The notion of sociability allows catching the coherence of Maurice Blanchot's thought in the linking of the different points of his works. From the loneliness, which means in literature an exteriority from every other human activity, appears the communication, the way of being of literary work, outside of the values and outside of the world (social, political). The notions of friendship and of community developed by Blanchot are thought on this model as relationships between writers, who experienced this outside and so can enter in links without domination issues which legitimate the intellectual commitment but imply an assumed leaving of the literary space.
\end{abstract}

Keywords : loneliness and writing, friendship, community, intellectuals, political commitment and literature

Mots clés : solitude et écriture, amitié littéraire, communauté en littérature, intellectuels, engagement politique et littérature

De Maurice Blanchot, on répète à l'envi qu'il est l'écrivain du retrait. La formule est devenue sa fonction au panthéon de la littérature française. Elle témoigne de la perception idéale d'un auteur-ermite, invisible (aussi bien médiatiquement que physiquement) dont l'existence aura été « vouée à la littérature et au silence qui lui est propre ${ }^{1} »$, silence que certains ne manquent pas de trouver complaisant parce qu'ils se complaisent à le rappeler sans cesse. Derrière la sentence, il y a surtout le fantasme d'une radicale et exigeante adéquation entre une pensée, plaçant la solitude au centre de la littérature et en particulier de l'expérience de l'écriture, et d'une vie éclairant et justifiant cette pensée. Tout ceci n'est pas faux. Et pourtant, comme l'écrit Christophe Bident: «le retrait ne signifie

1. Formule biographique présentée dans les ouvrages de Maurice Blanchot republiés en poche. 
pas la réclusion ${ }^{2} »$. Car la vie de Blanchot fut également marquée par un engagement sans faille à des moments clés de l'histoire, par des amitiés, parties prenantes dans la construction de sa propre pensée (Bataille et Levinas en tête), ainsi que par un nombre important d'articles politiques. Cela, personne ne songe non plus à le nier.

Les deux discours cohabitent tant bien que mal, la plupart du temps, ils se superposent, rarement ils se rencontrent. Les unifier comme les dissocier semble insatisfaisant, l'un parce qu'il efface la spécificité de chacune des notions en l'assimilant aux autres; l'autre parce qu'il traite chaque aspect indépendamment et ne parvient ainsi qu'à construire une succession de figures : Blanchot écrivain du retrait, Blanchot théoricien de l'amitié et de la communauté, Blanchot intellectuel... renonçant à son tour à la spécificité d'une pensée qui les contient toutes et, plus grave, ouvrant la voie à l'accusation d'un « double langage ${ }^{3}$ », c'est-à-dire au mieux à une incohérence grave, au pire à une escroquerie de la pensée.

Pour tenter de retrouver l'unité de la pensée blanchotienne, un pas de côté est nécessaire, nécessaire aussi de sortir du vocabulaire blanchotien dans ce qu'il peut avoir de fascinant, pour mieux y retourner sous un éclairage nouveau. La notion de sociabilité est étrangère à l'œuvre de Blanchot. En un sens leur association est même scandaleuse car trop inadéquate. Il ne s'agit bien entendu pas d'y chercher un quelconque tableau sociologique du monde des lettres mais, en gardant le sens générique du terme recouvrant l'ensemble des relations entre les membres d'un groupe ${ }^{4}$, de l'utiliser comme cadre général d'une interrogation nouvelle de notions bien connues des lecteurs de Blanchot : la solitude, la communication, l'amitié, la communauté, sous un angle qui tente d'en rétablir les liens et les ruptures désormais pensées comme telles.

\section{«Ce que voudrait désigner le mot solitude ${ }^{5}$ »}

La solitude structure l'ensemble des expériences de la littérature. Tous, auteur, lecteur et œuvre, doivent l'accepter. Elle est cependant bien loin de ce que le sens commun entend par le terme. Dans L'Espace littéraire, tout entier placé sous les auspices de la solitude, Blanchot prend bien soin de

2. Christophe Bident, Maurice Blanchot. Partenaire invisible, Seyssel, Champ Vallon, 1998, p. 301.

3. La critique est formulée clairement par Henri Meschonnic : «Blanchot a deux langages. Selon les deux ordres du politique et du poétique, ni le discours ni les postulats ne sont les mêmes. » Henri Meschonnic, Poésie sans réponse, Paris, Gallimard, coll. " Le chemin », 1978, p. 81.

4. Qui est la définition du dictionnaire. Le groupe étant en l'occurrence le « monde » de la littérature, et en particulier structuré par l'espace littéraire; les membres étant ici l'auteur, le lecteur et l'œuvre.

5. Maurice Blanchot, L'Espace littéraire [1955], Paris Gallimard, coll. « Folio », 1988, p. 13. 
distinguer celle « essentielle » de la littérature de celle qui a lieu « dans le monde ». Cette dernière, héritée de la pensée d'Heidegger, part du postulat selon lequel le « Je suis ", comme affirmation de l'individu en tant que créature sociale, est en rupture avec ce que l'être peut avoir de lien avec la nature. Or cette dissimulation de l'être, si elle devient consciente, peut se changer en " séparation des êtres : l'absolu d'un Je suis qui veut s'affirmer sans les autres ${ }^{6} \|$, engendrant deux perceptions opposées liées à cette découverte. La première, ressentie comme une malédiction où « Je suis découvre le néant qui le fonde », devient incapacité à jouer le jeu social et se traduit par une exclusion; la seconde, elle, est perçue comme une puissance sans borne du « Je suis » : « L'orgueil d'une maitrise solitaire, la culture des différences, le moment de la subjectivité, qui brise la tension dialectique par laquelle elle se réalise ${ }^{7}$. » Cependant et quelle que soit la solitude ainsi engendrée, elle n'en demeure pas moins toujours « au niveau du monde », née de la société et se pensant, même comme séparation, irrémédiablement en rapport avec l'expérience commune qui la fonde.

Qu'est-ce qui rend alors 1'autre solitude « essentielle»? Précisément l'absence de ces liens «au monde». La solitude ici, n'est plus la conséquence d'une séparation - acte social - d'avec l'être, mais à la fois l'acte fondateur, la manifestation et la substance d'un « être » autre, de pur langage, et qui en ceci ne dépend en rien d'une existence commune :

La solitude de l'œuvre a pour premier cadre cette absence d'exigence qui ne permet jamais de la dire achevée ou inachevée. Elle est sans preuve, de même qu'elle est sans usage. Elle ne se vérifie pas, la vérité peut la saisir, la renommée l'éclaire : cette existence ne la concerne pas, cette évidence ne la rend ni sûre ni réelle, ne la rend pas manifeste ${ }^{8}$.

La littérature est donc irrémédiablement incertaine, insaisissable, indéfinissable, surtout à partir du monde. En ceci, elle n'est pas, sans risque de perdre sa substance, « utilisable ». Plus encore en dehors d'elle-même, comme œuvre et comme solitude, elle n'exprime rien, ne dit rien; ce qu'un regard social y lit, elle ne le dit pas, ne peut pas le dire.

Derrière le terme de solitude, comme ensuite et d'une manière toute différente, derrière le « neutre », ce qui s'exprime est une extériorité absolue de la littérature au monde, si absolue qu'elle ne peut ni s'exprimer ni a fortiori être exprimée par un langage autre, y compris celui de la critique?

6. Ibid., p. 338. Voir plus largement l'ensemble de l'annexe « La solitude essentielle et la solitude dans le monde $»$.

7. Ibid.

8. Ibid., p.15.

9. Dans le même mouvement néanmoins, cette tentative «d'écrire sur l'impossibilité d'écrire " (Roger Laporte) fonde toute la démarche critique blanchotienne et détermine en grande partie sa forme. On peut par exemple se risquer à avancer l'hypothèse que les nombreux oxymores dont Blanchot fait l'usage, loin d'être gratuits, expriment en fait le 
Car le neutre « refuse l'appartenance aussi bien à la catégorie de l'objet qu'à celle du sujet. Et cela ne veut pas dire qu'il est indéterminé et comme hésitant entre les deux, cela veut dire qu'il suppose une relation autre, ne relevant ni des conditions objectives, ni des dispositions subjectives ${ }^{10} »$. Ainsi exprimé, le neutre, invérifiable, impersonnel et indéfini par nature, indiquant néanmoins cet inconnu qu'il ne peut " dévoiler » est bien cette « relation autre », trouvant son origine dans le langage devenu littérature dont il est le fruit ${ }^{11}$.

Car cette solitude - ce qui la rend essentielle - se situe dans le langage, et plus précisément dans cette opération qui transforme le langage en littérature. Cette transformation puise en premier lieu son origine dans ce que Blanchot, questionnant à la suite d'Alexandre Kojève une idée hégélienne, appelle la puissance de négativité du langage que résume la phrase : «Le mot me donne l'être, mais il me le donne privé d'être ${ }^{12}$. » Derrière la formule se trouve l'idée que :

Tout notre langage - et c'est là sa nature divine - est agencé pour révéler, en ce qui « est », non ce qui disparaît, mais ce qui toujours subsiste et en cette disparition se forme : le sens, l'idée, l'universel; ainsi ne retient-il de la présence, que ce qui échappant à la corruption, marque et sceau de l'être (sa gloire aussi), n'est donc pas vraiment non plus ${ }^{13}$.

Autrement dit, le langage remplaçant le particulier de l'entité matérielle désignée, son hic et nunc ${ }^{14}$, par le générique du concept, la nie dans le même mouvement qu'elle l'exprime. Cette puissance de négation porte de ce fait la possibilité de la mort de l'entité particulière, qu'elle annonce en la détachant d'elle-même. De cet acte de dissociation découle néanmoins que le langage ne désignera que de manière imparfaite l'être, se situant de ce fait à la fois «au-delà » et « en deçà » de la simple représentation.

Cela établi, une nuance s'impose. Tous les langages ne sont pas équivalents face à cette puissance de négation. Reprenant cette fois une réflexion esquissée par Mallarmé, Blanchot pense le langage dans un double état,

caractère radicalement non dialectique de ce rapport autre engendré par le langage littéraire, les mots ne renvoyant à aucune réalité, ne sauraient en effet exprimer une quelconque idée sûre car démontrable.

10. Maurice Blanchot, L'Entretien infini, Paris, Gallimard, 1969, p. 440.

11. «Le neutre n'est d'ailleurs pas pensable, pointe à l'extrémité de la pensée et cette pensée, Blanchot l'a davantage écrite que théorisée. » Christophe Bident, op. cit., p. 437.

12. Maurice Blanchot, «La littérature et le droit à la mort », La Part du feu, Paris, Gallimard, 1949, p. 349.

13. Maurice Blanchot, L'Entretien infini, op. cit., p. 47.

14. Alexandre Kojève, Introduction à la lecture d'Hegel, Paris, Gallimard, coll. « Tel», 1947, p. 542. 
ou pour mieux dire en $"$ deux versions ${ }^{15}$ ", la première « quotidienne ", " courante » et la seconde " poétique ", « littéraire » ou " de la fiction », correspondant, même s'il récuse partiellement les termes, au « double état de la parole, brut ou immédiat ici, là essentiel ${ }^{16} \gg$ de Mallarmé. Ces deux états, s'ils naissent du même acte de dissociation avec le réel, divergent par leur destination. Car là où le langage courant, englobant aussi bien le bavardage quotidien que les œuvres scientifiques, justifié par son utilité concrète, disparaît dans sa fonction, devient simple signe du monde, et y retourne donc par son usage, la parole littéraire, entérinant la dissociation entre langage et monde qui la fonde, se «niche » dans l'intervalle qui les sépare, tirant du langage sa seule existence, et créé ainsi son propre espace, celui d'un langage clos sur lui-même n'exprimant que lui-même. Dès lors, comme le souligne Michel Foucault : «En fait, l'événement qui a fait naître ce qu'au sens strict on entend par "littérature" n'est de l'ordre de l'intériorisation que pour un regard de surface; il s'agit plutôt d'un passage au "dehors" $[\ldots]^{17} \gg$, c'est-à-dire non pas manifestation d'une subjectivité (celle de l'auteur), pas plus que d'une objectivité (la vérité «l'œuvre découvre, d'une découverte qui n'est pas vérité ${ }^{18} \gg$ ) mais d'une impersonnalité radicale, celle du neutre, ne tolérant aucune remise en question, transformant son extériorité substantielle en manifestation d'une " solitude » qu'il convient bien de nommer essentielle.

De cette conception découle logiquement une « contamination » de la solitude de l'œuvre à celui qui s'en approche, qu'il soit auteur ou lecteur. Car si le " dehors » est incompatible avec le monde, si en outre l'œuvre, produit et productrice de celui-ci, ne peut s'en dissocier, auteur et lecteur doivent de fait passer sous son influence : «L'œuvre est solitaire : cela ne signifie pas qu'elle reste incommunicable, que le lecteur lui manque. Mais qui la lit entre dans cette affirmation de la solitude de l'œuvre, qui l'écrit appartient au risque de cette solitude ${ }^{19}$. $\gg$ Cependant cette expérience n'est que superficiellement commune. La nuance ici est de taille : le lecteur en " entrant » dans l'affirmation, garde un rôle « actif », et plus encore une liberté implicite, celle de ne pas lire, qui fonde le détachement et la légèreté auxquels Blanchot attache toujours la lecture; l'écrivain à l'inverse, n'est pas libre, au sens strict, il « appartient », par l'acte d'écrire, à la sphère

15. Anne-Lise Schulte-Nordholt, Maurice Blanchot. L'écriture comme expérience du dehors, Genève, Droz, 1995. Concernant cette théorie du langage chez Blanchot, ici rapidement tracée, voir le premier chapitre de l'ouvrage intitulé « Le double état du langage ». Voir également du même auteur "Le vrai bilinguisme », Europe, n 940-941, août-septembre 2007, p. 85-100.

16. Stéphane Mallarmé, « Crise de vers », dans Euvres complètes, vol. 2, Paris, Gallimard, coll. «Bibliothèque de la Pléiade », 2003, p. 212.

17. Michel Foucault, « La pensée du dehors », Critique, n 229, juin 1966, p. 524.

18. Emmanuel Levinas, Sur Maurice Blanchot, Montpellier, Fata Morgana, 1975, p. 22.

19. Maurice Blanchot, L'Espace littéraire, op. cit., p. 15. 
d'influence du « dehors ». Cette différence fondamentale dessine le rôle de chacun. Ainsi, si le lecteur est, dans la pensée blanchotienne, une figure de la communication, l'écrivain quant à lui est sans conteste celle de la solitude.

L'expérience de l'écriture, pour l'écrivain, est en bien des sens un apprentissage de la solitude :

Qui creuse le vers, échappe à l'être comme certitude, rencontre l'absence des dieux, vit dans l'intimité de cette absence, en devient responsable, en assume le risque, en supporte la faveur. Qui creuse le vers doit renoncer à toute idole, doit briser avec tout, n'avoir pas la vérité pour horizon, ni l'avenir pour séjour, car il n'a nullement droit à l'espérance : il lui faut au contraire désespérer. Qui creuse le vers, meurt, rencontre sa mort comme abîme ${ }^{20}$.

Celui qui écrit épuise le monde en lui, devient étranger à ses valeurs, vérités, croyances ou espérances n'ayant plus aucun sens dans le monde désolé du langage littéraire. Il s'agit donc ici d'une expérience éminemment tragique, débouchant sur l'abîme de la mort, moins une épreuve finalement qu'un sacrifice sans consolation possible. En ceci :

L'expérience n'est pas l'expérience d'une certaine chose et certes pas de la littérature comme chose, mais la pure épreuve qui ouvre et véhicule en elle-même son propre champ. Écrire est perdre pied. Peut-être faudrait-il ajouter qu'un tel « perdre pied» ne s'accomplit que par l'écriture ${ }^{21}$.

Ce «perdre pied» en outre ne se limite pas à un seul renversement des valeurs. Plus total et plus violent, il appelle une disparition de l'auteur comme sujet, entérinant l'analogie omniprésente entre écriture et mort :

L'œuvre demande cela que l'homme qui l'écrit se sacrifie pour l'œuvre, devienne autre, devienne non pas un autre, non pas du vivant qu'il était, l'écrivain avec ses devoirs, ses satisfactions et ses intérêts, mais plutôt personne, le lieu vide et animé où retentit l'appel de l'œuvre ${ }^{22}$.

L'expérience de l'écriture, pour l'écrivain, s'achève - et en même temps commence réellement - avec cette perte d'identité ${ }^{23}$. Créé par l'œuvre pour être le réceptacle vide de son écriture (de son inscription), l'écrivain

20. Ibid., p. 37-38.

21. Françoise Collin, Maurice Blanchot et la question de l'écriture, Paris, Gallimard, coll. « Tel », 1971, p. 29-30.

22. Maurice Blanchot, Le Livre à venir [1959], Paris, Gallimard, coll. «Folio », 1986, p. 293.

23. Cette disparition, Blanchot la voit dans plusieurs aspects, en particulier dans le passage $\mathrm{du}$ « Je » au « Il », comme passage au neutre, ou encore, dans Le Pas au-delà, par le statut particulier du nom de l'écrivain qui, désormais attaché à l'œuvre, ne renvoie plus à lui comme personne et signe son absence du monde. 
se doit de garder le silence : «Je rends sensible, par ma médiation silencieuse, l'affirmation ininterrompue, le murmure géant sur lequel le langage en s'ouvrant devient image, devient imaginaire, profondeur parlante, indistincte plénitude qui est vide ${ }^{24}$. » Le rôle de l'écrivain est de disparaître, son mode d'action est le silence, seul élément qui reste pleinement sien.

Là sans doute se situe le problème. Car si l'écrivain est appelé, soumis à la passivité, cela ne signifie pas qu'il accepte cette condition, pourtant sine qua non, de la création authentiquement littéraire. Au contraire, c'est comme un rapport de force incessant, quoique déséquilibré, entre l'œuvre naissante et l'écrivain, à qui est dénié le titre d'auteur mais qui pour autant ne se résout pas à disparaître :

[...] ce qui parle n'est plus lui-même, mais n'est pas non plus le pur glissement de la parole de personne. Du « Je » effacé, il garde l'affirmation autoritaire, quoique silencieuse. Du temps actif, de l'instant, il garde le tranchant, la rapidité violente. Ainsi se préserve-t-il à l'intérieur de l'œuvre, se contient-il là où il n'y a plus de retenue. Mais l'œuvre garde aussi, à cause de cela un contenu, elle n'est pas toute intérieure à elle-même ${ }^{25}$.

Combat sans vainqueur possible, mais inévitable cependant. L'œuvre ne peut exister sans quelqu'un qui l'écrive, l'écrivain ne peut se considérer tel tant qu'aucune œuvre ne le prouve; l'œuvre a besoin qu'il disparaisse mais sa disparition empêcherait l'achèvement de l'écriture. L'œuvre, de ce fait nécessairement imparfaite au regard des exigences du « dehors", rend alors la «pure» littérature, sans concession au monde, éternellement « à venir».

La perte de repères, le progressif sentiment d'étrangeté que l'écrivain ressent face au monde et qui en un sens signe sa mort sous l'influence du « dehors », ne signifie pas pour autant qu'il en est résident : « Celui qui écrit l'œuvre est mis à part, celui qui l'a écrite est congédié26. » Au mouvement de séparation du monde correspond un autre symétrique qui rejette l'écrivain de l'œuvre en gestation. Exclusion d'autant plus inévitable qu'elle est primordiale à son achèvement, qui n'est pas dans le pouvoir de l'écrivain de réaliser :

L'écrivain écrit un livre, mais le livre n'est pas encore l'œuvre, l'œuvre n'est œuvre que lorsque se prononce par elle, dans la violence d'un commencement qui lui est propre, le mot être, événement qui s'accomplit quand l'œuvre est l'intimité de quelqu'un qui l'écrit et de quelqu'un qui la lit $^{27}$.

24. Maurice Blanchot, L'Espace littéraire, op. cit., p. 22.

25. Ibid.

26. Ibid., p. 14.

27. Ibid., p. 15. 
Dépossédé du commencement de l'œuvre, l'écrivain l'est aussi de son achèvement, placé sous la sentence maintes fois réitérée par Blanchot: Noli me legere. Impossibilité et interdiction pour l'écrivain de se lire qui rend nécessaire la médiation d'un lecteur, anonyme et indéterminé par essence, «n'importe quel lecteur, unique, mais transparent ${ }^{28}$ ». Son intervention est alors aussi voire plus créatrice de l'œuvre ${ }^{29}$ que celle de l'écrivain, par l'autonomie qu'elle lui donne face à la présence de ce dernier qui ne veut pas disparaître, permettant ainsi à cette œuvre d'acquérir l'impersonnalité sans laquelle elle ne peut devenir littérature.

\section{Espace littéraire et communication}

Avec la lecture, par le «déploiement» de l'œuvre qu'elle permet, s'introduit la question de la communication. Son intervention est invisible. Mais c'est là précisément son rôle :

La lecture ne fait rien, n'ajoute rien; elle laisse être ce qui est; elle est liberté, non pas liberté qui donne l'être ou le saisit, mais liberté qui accueille, consent, dit oui, ne peut que dire oui et, dans l'espace ouvert par ce oui, laisse s'affirmer la décision bouleversante de l'œuvre, l'affirmation qu'elle est - et rien de plus ${ }^{30}$.

Car pour Blanchot peu importe qui lit l'œuvre, peu importe surtout ce qu'il en comprend, son interprétation ne concerne pas l'œuvre, au contraire c'est sa transparence, sa superfluité, qui permet l'association sinon impossible entre la solitude essentielle de la littérature et la diffusion de l'œuvre, sa publicité ${ }^{31}$, fondatrice d'un intervalle qui lui est propre, l'espace littéraire, et génératrice d'un nouveau mode de relation « hors du monde » : la communication.

L'espace littéraire est difficile à saisir. Notion fondamentalement polysémique, elle recouvre une multiplicité d'états que Blanchot conçoit comme complémentaires et non concurrents. Il recouvre ainsi d'abord (ou finalement) cet espace indépendant du monde qui est propre à l'œuvre une fois achevée. Dans une large mesure il désigne également le processus entier par lequel intervient cet achèvement, la spatialité remplaçant une chronologie en défaut dans le mode d'existence atemporel de la littérature.

\footnotetext{
28. Ibid., p. 254.

29. «Lire ce serait donc, non pas écrire à nouveau le livre, mais faire que le livre s'écrive ou soit écrit - cette fois sans l'intermédiaire de l'écrivain, sans personne qui l'écrive. » Maurice Blanchot, L'Espace littéraire, op. cit., p. 254.

30. Ibid., p. 255.

31. Dans Le Livre à venir Blanchot utilise également le terme « public », en lui donnant les mêmes attributs qu'au lecteur, à savoir indétermination, présence dans le monde qui permet paradoxalement à l'œuvre d'en sortir... Cela éclaire un peu plus sur le véritable rôle accordé à celui-ci.
} 
Il contient par là l'ensemble des relations auteur/lecteur/œuvre qui aboutit à l'avènement de la dernière; relations paradoxales s'il en est car elles constituent un double rapport d'interdépendance et d'exclusion mutuelle. L'interdépendance, déjà évoquée, semble évidente : il ne peut avoir d'œuvre si celle-ci n'est pas écrite (couchée sur le papier) par un écrivain et, rajoute Blanchot, si elle n'est pas lue par un lecteur. Mais à cette configuration s'ajoute une seconde qui en est le reflet : sans œuvre, il ne peut avoir ni écrivain ni a fortiori lecteur : "Il est du lecteur comme du poète. Tous deux, poète et lecteur reçoivent [du poème] leur existence et sont fortement conscients de dépendre, dans leur existence, de ce chant à venir, de ce lecteur en devenir ${ }^{32}$. $\gg$ Exprimée ainsi, cette théorie a tout d'une aporie. Pour comprendre l'articulation de ces deux jeux de dépendance, il faut percevoir comment s'entremêlent deux plans d'existence de l'œuvre. Le premier est pure potentialité, l'œuvre « existe » parce qu'elle contient les conditions qui président à sa future venue au monde, d'abord auprès de l'écrivain, comme « inspiration »: «L'inspiration ne signifie rien d'autre que l'antériorité du poème par rapport au poète, ce fait que celui-ci se sent, dans sa vie et dans son travail encore à venir, encore absent en face de l'œuvre poétique qui est elle-même tout avenir et toute absence ${ }^{33} »$, ensuite auprès du lecteur: "Que la poésie puisse se passer d'être lue, et que le poème doive ignorer orgueilleusement le lecteur, cela est faux, mais dans la mesure où, antérieur à lui, c'est justement le rôle du poème de préparer, de mettre au monde celui qui doit le lire $[\ldots]^{34} \gg$. En ceci, auteur et lecteur sont « tous deux uniques : n'ayant d'existence que par cette œuvre et à partir d'elle ${ }^{35}$ », en ceci aussi ils meurent dans sa réalisation, puisque l'agencement qui les fait naître n'a lieu qu'une fois. C'est en ce sens, que l'écriture de l'œuvre est pour Blanchot un « recommencement », toujours précédée par cette existence antérieure prenant son origine dans le « dehors» de la littérature. Origine irrémédiablement inconnue, qu'elle ne cesse de chercher à retrouver dans l'écriture et la lecture qui, conditions de sa réalisation, de sa re-naissance, sont aussi des bornes irréductibles à son accomplissement et à son retour vers cette origine ${ }^{36}$.

32. Maurice Blanchot, La Part du feu, op. cit., p. 104.

33. Ibid.

34. Ibid., p. 103.

35. Maurice Blanchot, L'Espace littéraire, op. cit., p. 302.

36. Cette question de l'origine de l'œuvre, comme ce qui la fonde en tant qu'œuvre de littérature, de son essence littéraire, Blanchot la puise en grande partie chez Heidegger, en particulier dans L'Origine de l'cuvre d'art. Chez les deux on retrouve ainsi l'exposé de la non-primauté de l'artiste sur l'œuvre, l'idée que l'art n'est pas mimésis de la réalité des choses, de leur " choséité ». Les deux pensées divergent néanmoins quant à la nature de cette origine. Pour Heidegger, l'essence/origine de l'art est la " vérité », l'art étant dévoilement d'une vérité du monde alors que pour Blanchot art et monde ne sauraient être en rapport, la littérature étant toujours au-delà et en deçà de toute vérité, et en cela un éternel non-vrai, ce que traduit, entre autres choses, le neutre. 
L'espace de l'œuvre «achevée », débarrassée du poids de l'auteur par l'intervention du lecteur, engendre un nouveau rapport qui reflète son passage au dehors, la communication. En ce sens la configuration des relations produites par l'espace littéraire (et le produisant dans un mouvement simultané) est résolument paradoxale puisque, tout en étant tributaire d'une interdépendance auteur-lecteur-œuvre, elle ne s'établit que sur leur exclusion mutuelle et progressive jusqu'à ce qu'il n'en reste que ce substrat qu'est l'œuvre littéraire. Pour cette raison le type de « sociabilité » unissant lecteur et auteur ne peut lui-même qu'être paradoxal : «L'écrivain aime dire qu'il écrit son livre en le destinant à l'unique ami. Vœu bien déçu. Dans le public, l'ami n'a pas de place ${ }^{37}$.» Ce passage ne laisse aucune ambiguïté. Si l'amitié existe, elle ne peut en aucun cas unir auteur et lecteur. D'autant plus que la puissance d'arrachement de l'auteur à son œuvre, qui fait de la lecture, et non de l'écriture, la part « créative » est ressentie avec toute sa violence par celui-ci, comme le décrit longuement Blanchot dans « La littérature et le droit à la mort »: «L'auteur voit les autres s'intéresser à son œuvre, mais l'intérêt qu'ils y portent est un intérêt autre que celui qui avait fait d'elle la pure traduction de lui-même, et cet intérêt autre change l'œuvre, la transforme en quelque chose d'autre où il ne reconnaît pas la perfection première ${ }^{38}$. » Dès lors et si dans sa double solitude le lecteur peut faire office, faute de mieux, de « compagnon » de l'auteur, les relations entre les deux figures sont éminemment conflictuelles :

Les rapports noués par l'auteur avec le lecteur, rapports que je qualifierai d'étranglement, où chacun, sans en avoir l'air et avec une politesse froide, tient l'autre à la gorge, sont d'abord des rapports de l'auteur avec lui-même, un moyen pour lui de se voir, tel qu'il se verrait si, au lieu d'écrire, il lisait et, lisant, se lisait lui-même. Mais cela ne se peut $\operatorname{pas}^{39}$.

Le lecteur, ici encore à venir, double de l'écrivain écrivant, est donc, dans le même temps, la personnification (impersonnelle) de l'impossibilité qu'il a de se lire lui-même, du Noli me legere, et porte par là sa négation future, la « réécriture » de l'œuvre sans lui, condition d'émergence de la communication :

Écrire, la demande d'écriture (non plus l'écriture qui était toujours mise au service de la parole orale ou encore de la pensée idéologique mais, au contraire, l'écriture doucement libérée par sa propre force comme si elle s'adonnait à l'interrogation qu'elle est la seule à cacher) libère peu à peu toutes les autres possibilités, une manière anonyme d'être en relation et de communiquer (qui remet tout en question, en premier lieu l'idée sur Dieu, sur la vérité et ensuite sur le Livre et l'(Euvre eux-mêmes) afin que cette écriture considérée dans son austérité énigmatique n'ait pas pour finalité le

37. Maurice Blanchot, Le Livre à venir, op. cit., p. 334.

38. Maurice Blanchot, La Part du feu, op. cit., p. 265.

39. Maurice Blanchot, L'Amitié, Paris, Gallimard, 1971, p. 143. 
Livre, la marque de la fin en quelque sorte, mais l'écriture que l'on pourrait envisager hors du discours, hors du langage ${ }^{40}$.

La communication, cette « manière anonyme d'être en relation », qui est le propre de l'œuvre désormais solitaire, n'est cependant aucunement communication de quelque chose : « La communication de l'œuvre, n'est pas dans le fait qu'elle est devenue communicable, par la lecture, à un lecteur. L'œuvre est elle-même communication $[\ldots]^{41} \gg$. Elle est alors bel et bien un rapport « hors de tout rapport», non seulement libre de tout lien au monde, mais aussi, de par sa simple existence, porteuse d'une colossale puissance de contestation de ses valeurs (Dieu, la Vérité...), contestation $^{42}$ radicale et infinie de tout pouvoir, « lieu où le langage est encore relation sans pouvoir, langage du rapport nu, étranger à toute maîtrise et à toute servitude ${ }^{43} \gg$. Cette communication, interne à l'œuvre, se dédouble en mouvements symétriques infinis de recherche de l'origine d'une part, et de tentative d'accomplissement d'autre part, où l'œuvre interroge son essence littéraire à travers elle-même et toutes les autres œuvres passées comme futures, sans réponse possible.

Mais la communication engendre un autre développement. «Contaminant » le monde, elle laisse entrevoir à ceux qui ont subi l'influence du « dehors », la possibilité d'une relation similaire :

La « communication » telle qu'elle se dévoile dans les rapports humains privés et se retire dans les œuvres que nous appelons encore œuvres d'art, ne nous indique peut-être pas l'horizon d'un monde dégagé des rapports trompeurs, mais nous aide à récuser l'instance qui fonde ces rapports, nous forçant à gagner une position où il nous serait possible de n'avoir pas de part aux « valeurs » ${ }^{44}$.

Ainsi, et dans un mouvement analogue à celui de l'œuvre : « Ce n'est que lorsque l'homme se sera accompli (supprimé comme pouvoir) que la relation à l'homme cessera elle-même d'être un pouvoir et deviendra relation possible, "communication" ${ }^{45}$. " C'est seulement à partir de cette possibilité que s'ouvrent et peuvent se comprendre les nouveaux rapports émergeants, portés par l'horizon d'un « communisme de pensée », à savoir l'amitié et la communauté.

40. Maurice Blanchot, Écrits politiques (1953-1993), Paris, Gallimard, coll. « Les cahiers de la $N R F », 2008$.

41. Maurice Blanchot, L'Espace littéraire, op. cit., p. 263.

42. Cette idée d'une puissance infinie de contestation et de refus de la littérature, Blanchot la partage avec Georges Bataille notamment, explicitement, dans l'avant-propos de La Littérature et le Mal.

43. Maurice Blanchot, Le Livre à venir, op. cit., p. 49.

44. Maurice Blanchot, L'Amitié, op. cit., p. 113.

45. Ibid., p. 110. 


\section{L’amitié, au-delà de la solitude de l'écrivain?}

On ne peut dissocier, chez Blanchot, l'amitié de la littérature. Non pas que celle-ci concerne l'œuvre ou y retourne comme le fait la communication mais parce qu'elle naît directement, pour l'écrivain, de l'expérience du « dehors ». En effet, la « double» solitude de l'auteur, produit de son incapacité à disparaitre dans l'écriture et tout autant à réintégrer un monde dont il ne comprend plus les valeurs, fait de lui un être en tout point singulier car né de l'écriture d'une œuvre spécifique. Cet état de « désœuvrement », Blanchot le questionne constamment dans ses livres, y compris dans des fragments « narratifs », comme celui ouvrant L'Entretien infini : « Ô neutre, libère-moi de ma fatigue, conduis-moi vers cela qui, quoique me préoccupant au point d'occuper toute la place, ne me concerne pas. / Mais c'est cela la fatigue, un état qui n'est pas possessif, qui absorbe sans mettre en question ${ }^{46}$. » Or, cette fatigue, conséquence du contact avec le neutre, manifestation de la solitude de l'écrivain, est ce qui sert de terreau à l'amitié :

Il m'est venu cette pensée, c'est bien qu'il n'y a pas d'autre raison à votre amitié - et combien elle est assidue, désintéressée, je ne saurais assez le dire - que ce que j'ai de plus particulier et qui est ma part privilégiée. Mais peut-on s'attacher à un homme fatigué en raison seulement de sa fatigue? « Je ne demande pas qu'on supprime la fatigue. Je demande à être reconduit dans une région où il soit possible d'être fatigué ${ }^{47}$. "

C'est en cela que l'on peut considérer le concept blanchotien d'amitié comme indissociable d'une expérience de la solitude née de l'écriture et du désœuvrement qui frappent l'écrivain. Pour cette raison, il est difficile de penser ce rapport hors d'un cadre proprement littéraire, du moins dans ces conditions d'émergence :

L'amitié n'est pas un don, une promesse, la générosité générique. Rapport incommensurable de l'un à l'autre, elle est le dehors relié dans sa rupture et son inaccessibilité. Le désir, pur désir impur, est l'appel à franchir la distance, appel à mourir en commun par la séparation ${ }^{48}$.

L'amitié se pense donc avant tout comme reconnaissance de l'altérité inaliénable de l'ami, prenant ainsi sa source non pas dans ce qui lie soi-même et autrui comme semblables, mais au contraire dans l'acceptation de leur séparation, qui est aussi commune distance face au monde. Blanchot s'oppose dès lors à la tradition grecque de la philia, tout en soulignant son rôle de «modèle de ce qu'il y a d'excellent dans les relations humaines ${ }^{49} \gg$. Il en nie pourtant les postulats :

46. Maurice Blanchot, L'Entretien infini, op. cit., p. XXI.

47. Ibid., p. XX.

48. Maurice Blanchot, L'Écriture du désastre, Paris, Gallimard, 1980, p. 50.

49. Maurice Blanchot, « Michel Foucault tel que je l'imagine», dans Une voix venue d'ailleurs, Paris, Gallimard, coll. « Folio », 2002, p. 152. 
La philia grecque est réciprocité, échange du Même avec le Même, mais jamais ouverture à l'Autre, découverte d'Autrui en tant que responsable de lui-même, éveil et dégrisement par cet Autrui qui ne me laisse jamais tranquille, jouissance (sans concupiscence, comme dit Pascal) de sa Hauteur, de ce qui le rend toujours plus près du Bien que $«$ moi $»{ }^{50}$.

Ce respect de l'altérité de l'ami fait de l'amitié une «relation sans pouvoir », identique en cela à ce qu'est la communication au regard de l'œuvre, c'est-à-dire un rapport non social où autrui, plus pensé par rapport à soi, est accepté dans sa pleine altérité :

[...] le pur intervalle qui, de moi à cet autrui qu'est un ami, mesure tout ce qu'il y a entre nous, l'interruption d'être qui ne m'autorise jamais à disposer de lui ni de mon savoir sur lui (fût-ce pour le louer) et qui loin d'empêcher toute communication, nous rapporte l'un à l'autre dans la différence et parfois le silence de la parole ${ }^{51}$.

Il est tentant de voir en l'amitié un autre terme exprimant une relation similaire à la communication. Perçue ainsi, elle serait pour l'écrivain une nouvelle illusion l'encourageant à poursuivre ce « silence de la parole » qu'est l'écriture. Car, cette amitié ne semble pas réellement dissocier la pensée/écriture et celui qui la produit. Deux rapports se superposent en réalité. D'une part, une « amitié intellectuelle ${ }^{52}$ » qui est en fait reconnaissance ${ }^{53}$ de deux pensées « amies » entamant un dialogue, sur le modèle de la communication. Et d'autre part une amitié entre écrivains, « repersonnifiés », désormais indissociables de « leur» œuvre.

Là se trouve le point d'achoppement entre amitié et littérature. Car l'œuvre écrite par l'ami, redevient celle de l'ami, n'est plus placée dans cet anonymat qu'elle prend pour devenir authentique littérature. Le glissement sémantique entre œuvre et pensée effectué par Blanchot lorsqu'il parle de l'amitié et de ses amis est d'ailleurs évocateur de cette sortie d'un rapport purement littéraire. Par l'amitié, l'œuvre évoque à nouveau l'ami, témoigne de sa pensée; toutes choses que le processus littéraire, par la lecture, s'emploie habituellement à effacer :

Nous ne pouvons laisser de côté les sentiments d'amitié ni la tristesse. Et parler avec sang-froid d'ouvrages amis en ignorant l'ombre qui s'est retirée en eux et qu'ils jettent sur nous serait un mouvement sans vérité, du reste

50. Maurice Blanchot, Pour l'amitié [1996], Tours, Farrago, 2000, p. 33.

51. Maurice Blanchot, L'Amitié, Paris, Gallimard, 1971, p. 329. On peut y lire également à la même page : « Ce qui sépare : ce qui met authentiquement en rapport, l'abîme même des rapports où se tient avec simplicité, l'entente toujours maintenue de l'affirmation amicale. » 52. Maurice Blanchot, « Michel Foucault tel que je l'imagine », op. cit., p. 152. Le texte témoigne de la rencontre manquée entre les deux hommes qui n'empêche pas cette « amitié intellectuelle », posthume, pour l'œuvre et la pensée de Michel Foucault.

53. Autour de la question de la reconnaissance, voir Christophe Bident, Reconnaissances. Antelme, Blanchot, Deleuze, Paris, Calmann-Levy, 2003. 
hors de notre pouvoir. Que ces œuvres tout à coup nous manquent, il faut bien nous en convaincre, même si elles sont là, autour de nous, avec toutes les forces qui leur appartiennent. Ce manque ne les écarte pas de nous; il est la manière dont ils nous sont proches $[\ldots]^{54}$.

La présence de l'ami disparu ${ }^{55}$ se maintient dans son œuvre, empêchant son achèvement. L'oubli, nous dit Blanchot, finit toujours par effacer cette présence. Le frein qu'il donne à la littérature n'est donc que temporaire, et du reste seulement valable pour l'ami. Cela n'en éclaire pas moins les aboutissements extralittéraires de l'amitié entre écrivains, qui ouvrent la voie à un éloignement progressif pour ces derniers de l'espace du « dehors ».

\section{Ouverture sur le politique : communauté et intellectuel}

L'amitié ouvre une brèche entre la littérature et le monde que, sur son modèle, creuse encore la communauté. Les similitudes entre amitié et communauté sont nombreuses. Idées relativement tardives chez Blanchot, nées d'un dialogue avec la pensée de Bataille, elles partent toutes deux d'une même reconnaissance de l'altérité, d'un même refus d'assimilation d'autrui à soi, qui caractérise tous les rapports nés du modèle de la communication. En ceci la communauté n'est pas fusion, communion des membres dans une forme de « surindividualité » :

La communauté n'a pas à s'extasier, ni à dissoudre les éléments qui la composent en une unité surélevée qui se supprimerait elle-même en même temps qu'elle se supprimerait comme communauté. La communauté n'est pas pour autant la simple mise en commun, dans les limites qu'elle se tracerait, d'une volonté partagée d'être à plusieurs, fût-ce pour ne rien faire, c'està-dire ne rien faire d'autre que de maintenir le partage de « quelque chose » qui précisément semble toujours soustrait à la possibilité d'être considéré comme part à un partage : parole, silence ${ }^{56}$.

Il n'y a pas de communauté, donc, sans survivance des individus en elle, sans pour autant qu'elle se réduise à un seul regroupement d'intérêts.

Car ce qui la motive en premier lieu, c'est un partage existentiel, celui de la parole et du silence, qui explique pourquoi elle évoque tant de choses aux écrivains, mais surtout celui d'une impossibilité pour l'être de concevoir

54. Maurice Blanchot, L'Amitié, op. cit., p. 214.

55. L'amitié, pour Blanchot, est indissociable de la perte de l'ami. La mort, en effet, ne sépare pas les amis mais au contraire efface la séparation sur laquelle se fonde l'amitié, par le souvenir de la proximité et l'effacement des différends. En ce sens, et comme l'a très bien souligné Jacques Derrida, l'amitié est toujours rétrospective, consciente d'elle-même seulement lorsqu'elle n'est plus possible. Voir Jacques Derrida, Politiques de l'amitié, Paris, Galilée, 1994.

56. Maurice Blanchot, La Communauté inavouable, Paris, Minuit, 1983, p. 19. 
sa propre mort, qui rend nécessaire la relation à autrui : « la substitution mortelle est ce qui remplace la communion ${ }^{57} »$. En ce sens, la communauté établit un « hors de soi » commun, partage de ce qui ne se partage pas, lui donnant un caractère instable et incernable. De ce fait, comme l'a montré Jean-Luc Nancy, elle est aussi « désœuvrée », non productrice d'œuvre :

On ne la produit pas, on en fait l'expérience (ou son expérience nous fait), comme expérience de la finitude. La communauté comme œuvre, ou la communauté par les œuvres supposerait que l'être commun, comme tel soit objectivable et productible (dans des lieux, des personnes, des édifices, des discours, des institutions, des symboles : bref, dans des sujets) ${ }^{58}$.

Blanchot, pour cette raison, la définit essentiellement par le négatif (ce qu'elle n'est pas) ainsi que par les épithètes qui l'accompagnant la situent. Entre autres, il évoque 1'existence chez Bataille d'une idée de «communauté idéale de la communication ${ }^{59} »$, à laquelle on a parfois réduit la communauté blanchotienne. Cette dernière recouvre en fait la «sociabilité » qui se crée entre auteur, lecteur et œuvre, prototype de l'espace littéraire. On ne peut cependant assimiler les deux termes, car malgré la déférence dont il fait preuve envers son ami, Blanchot ne manque pas de pointer ce qu'ont d'incompatibles la communauté et le processus littéraire, en particulier autour du lecteur. Car la lecture doit être maintenue dans son anonymat, sans quoi elle est « lecture personnelle par des amis personnels ${ }^{60} »$, qui empêche la communauté de produire une œuvre. Ainsi, si une communauté se crée, entre l'ami qui écrit et l'ami qui lit, celleci ne saurait être autre chose qu'une « communauté d'écriture », rendant le lecteur coresponsable de ce qui est écrit, co-auteur dans le partage du temps de l'écriture qu'il détermine.

C'est ce que Blanchot conçoit comme la communauté de la revue, lorsqu'il porte l'idée d'une Revue internationale. Celle-ci non pas simple superposition de textes, se veut une véritable expérience communautaire :

\footnotetext{
Chacun devient responsable d'affirmations dont il n'est pas l'auteur, d'une recherche qui n'est pas seulement la sienne, il répond d'un savoir qu'il ne sait pas originellement par lui-même. C'est là le sens de la revue comme possibilité collective. C'est un statut intermédiaire entre auteur et lecteur ${ }^{61}$.
}

Ce passage montre bien, outre le statut particulier que prend la lecture dans l'espace communautaire de la revue (bien loin de celle attendue par l'espace littéraire), le mouvement par lequel la communauté constitue un

57. Ibid., p. 24.

58. Jean-Luc Nancy, La Communauté désœuvrée, Paris, Christian Bourgois, 1990, p. 79.

59. Maurice Blanchot, La Communauté inavouable, op. cit., p. 40.

60. Ibid., p. 45.

61. Maurice Blanchot, Écrits politiques (1953-1993), op. cit., p. 103. Voir l'ensemble des textes portant sur le projet de Revue internationale. 
« retour au monde » pour l'écrivain. Retour au monde, certes, mais qui s'appuie néanmoins sur son expérience d'écriture :

Cette revue ne sera donc pas une revue de culture : l'intérêt par exemple, que nous portons à la littérature n'est pas un intérêt de culture, quand nous écrivons, nous n'écrivons pas pour enrichir la culture générale. Ce qui nous importe, c'est une recherche de vérité ou encore une certaine exigence juste, peut-être de justice, pour laquelle l'affirmation, par son intérêt au centre, par son rapport unique au langage, est essentielle $\mathrm{e}^{62}$.

L'écriture en revue part donc de l'expérience littéraire. Mais, bien loin d'avoir la littérature pour but, elle détourne celle-ci, par l'expérience collective, à des fins éthique et politique (la vérité, la justice). Cette association fonde alors un « communisme de pensée », c'est-à-dire, sur l'image de la communication, une nouvelle relation sans pouvoir entre les membres ${ }^{63}$. En ce sens, la littérature disparaît, du moins comme finalité, se construisant encore de manière souterraine (son mode d'être), mais le glissement vers le politique, par la responsabilité publique, par le sens nouveau qu'y prend l'écriture, par l'intervention dans le monde des auteurs est largement entamé.

L'aboutissement de ce mouvement est la figure de l'intellectuel. Celleci, n'est en rien incompatible avec le reste de la pensée de Blanchot, car il n'entrevoit aucunement l'engagement comme intrinsèque à l'art qui, hors du monde, ne peut avoir d'impact sur lui : « L'art agit mal et agit peu [...] dès que l'art se mesure à l'action, l'action immédiate et pressante ne peut que lui donner tort, et l'art ne peut que se donner tort à lui-même ${ }^{64}$. » $\mathrm{Ce}$ n'est donc pas au nom de l'art que peut se faire l'engagement intellectuel de l'artiste. Au contraire, l'engagement n'est possible selon Blanchot que par une renonciation provisoire au statut d'écrivain, c'est-à-dire à soi-même :

Lorsque l'intellectuel - l'écrivain - se décide et se déclare, il subit un dommage irréparable. Il se soustrait à la seule tâche qui lui importe. Il se peut qu'il perde définitivement le droit à la parole inattendue. Entre deux nécessités qui s'imposent en ne s'imposant pas, il cède à celle pour laquelle il n'est pas fait ${ }^{65}$.

62. Ibid.

63. Blanchot, par l'influence de Dionys Mascolo, commence à penser ce «communisme », qui ne se trouve jamais réalisé en totalité par un régime ni accusé par les crimes éventuels de celui-ci. Toujours placé dans un idéal utopique de «relation sans pouvoir» entre les hommes, il est pris dans un rapport étrange de possibilité/impossibilité similaire à la création littéraire. Voir Maurice Blanchot, « Sur une approche du communisme », dans L'Amitié, op. cit., p. 109-114.

64. Maurice Blanchot, L'Espace littéraire, op. cit., p. 282.

65. Maurice Blanchot, Les Intellectuels en question. Ébauche d'une réflexion [1996], Tours, Farrago, 2000, p. 36. 
Or ce renoncement est un danger d'autant plus essentiel que l'engagement intellectuel se fait « pour répondre à des exigences morales à la fois obscures et impérieuses, puisqu'elles étaient de justice et de liberté ${ }^{66} "$. Poursuivi au nom de valeurs absolues, mais non permanent, l'engagement blanchotien ne correspond donc ni à un statut d'expert ("l'intellectuel spécifique » selon Michel Foucault, opposé à un « intellectuel universel ») ni à l'engagement de type sartrien, intrinsèque et largement partisan. La figure blanchotienne de l'intellectuel, au contraire, constitue un retour explicite aux « origines ", en l'occurrence à l'affaire Dreyfus, archétype d'un engagement au nom de principes universaux, mais pensé comme intervention ponctuelle dans la sphère publique ${ }^{67}$. Cela le condamne cependant à être une position défensive essentiellement en réaction aux événements, instaurant le « refus » comme fil conducteur principal.

Comment comprendre dès lors, la spécificité de l'engagement de l'écrivain? Précisément parce que son statut est ce qui le légitime. L'écrivain, s'engage depuis son retrait du monde, depuis sa solitude, car il voit les événements depuis le «dehors », depuis sa solitude. Mais l'engagement, motivé par le caractère exceptionnel des événements, rompt cet intervalle pour le replacer, au cœur du monde et de la vie publique. Ainsi, bien que se construisant sur son abolition, l'engagement intellectuel ne peut se légitimer sans cette spécificité antérieure de l'écrivain. En outre, ce retour au monde est aussi renoncement à la solitude essentielle qui marque le giron de l'œuvre, car l'action, par nature et pour aboutir nécessite le groupe : « Il est difficile d'être un intellectuel, d'autant plus qu'on ne saurait l'être seul et que les exigences personnelles qu'il semble être voué à porter (du moins en tant qu'il est écrivain) le confinent dans une solitude dont il ne peut se sortir indemne ${ }^{68}$. » Ainsi, l'engagement se pense essentiellement comme sociabilité mais aussi comme une expérience communautaire, structurée par une coresponsabilité de ses membres dans l'engagement et dans ses conséquences $^{69}$. L'écrivain est alors une nouvelle fois face à un paradoxe qui veut qu'il épouse pleinement sa condition d'écrivain, sinon toujours incertaine, au moment où il est appelé à y renoncer.

66. Ibid., p. 52.

67. L'engagement de Maurice Blanchot, après la guerre du moins, reflète cette conception, tant dans sa forme (pétitions, Le Manifeste des 121 surtout, déclaration de soutien pour Mandela, Kozovoï, rédaction de prospectus, etc.) que par son caractère ponctuel, alternant avec des périodes de « silence », et se polarisant autour de grands événements (le « coup d'État » gaulliste de 1958, la guerre d'Algérie, Mai 68, construction du mur de Berlin, etc.). 68. Maurice Blanchot, Les Intellectuels en question, op. cit., p. 32.

69. C'est le cas notamment du Manifeste des 121 où les intellectuels « ont aussi - et ce n'est pas le trait le moins significatif - fait l'expérience d'une manière d'être ensemble, et je ne songe pas seulement au caractère collectif de la Déclaration, mais à sa force impersonnelle, à ce fait que tous ceux qui l'ont signée lui ont certes apporté leur nom, mais sans s'autoriser de leur vérité particulière ou de leur renommée nominale ». Maurice Blanchot, Écrits politiques (1953-1993), op. cit., p. 96. 
Il n'y a donc pas chez Maurice Blanchot deux discours totalement distincts, mais tout au plus deux ordres l'un, le plus important, tourné vers la littérature et l'autre qu'on peut qualifier de « politique ». La différence est importante. S'il y a bien des ruptures dans la continuité de la pensée, elles ne se font aucunement en dépit de la cohérence de l'œuvre mais, tout au contraire, témoignent de celle-ci. Car à l'origine de tous les rapports envisagés par Blanchot se situe une solitude que seule l'expérience du " dehors » peut faire naître. Si l'amitié, la communauté et l'engagement se dissocient finalement de cette origine dans la recherche de leurs buts propres, ils ne doivent jamais leur existence, par extension ou imitation, qu'à la relation particulière et sans pouvoir qu'est la communication, produit de cette solitude essentielle de la littérature.

La «sociabilité » permet donc de comprendre la cohésion des différents thèmes blanchotiens, tout en révélant leurs agencements complexes, loin du simple agrégat de figures idéales. Cela, inversement, ne doit pas laisser croire à une linéarité de la pensée. L'univers qu'elle dessine n'est pas pacifié, tout est lutte continuelle, négation et exclusion mutuelles, qui pourtant ne sont pas les preuves de son inconsistance, mais bien celles de la rigueur, dans l'esprit de son auteur, des lois qui le régissent. Un univers, donc, pensé comme un tout, non plus accusé par sa multiplicité, mais la comprenant pleinement. 